
\title{
Resesacth Saute \\ On Board Pointing Error Detection and Estimation of Observation Satellite Data using Extended Kalman Filter
}

Dhanalakshmi R ( $\square$ dhanalakshmisaicse@outlook.com )

KCG College of Technology

\section{Eswara Chaitanya D}

RVR and JC College of Engineering

Chandra Shaker Reddy $P$

CMR College of Engineering and Technology

Vijendra Babu D

Aarupadai Veedu Institute of Technology

Puneet Kumar Aggarwal

ABES Engineering College

\section{Fantin Irudaya Raj E}

Dr Sivanthi Aditanar College of Engineering

\section{Research Article}

Keywords: Extended Kalman Filter, Jacobian matrix calculation, Linear expansion point, Onboard pointing error, Satellite data, System nonlinear degree

Posted Date: April 27th, 2021

DOl: https://doi.org/10.21203/rs.3.rs-446454/v1

License: (c) (i) This work is licensed under a Creative Commons Attribution 4.0 International License.

Read Full License 


\title{
ON BOARD POINTING ERROR DETECTION AND ESTIMATION OF OBSERVATION SATELLITE DATA USING EXTENDED KALMAN FILTER
}

\author{
${ }^{1}$ Dr.R.Dhanalakshmi, ${ }^{2}$ Dr. D. Eswara Chaitanya, ${ }^{3}$ P. Chandra Shaker Reddy, ${ }^{4}$ Dr.D.Vijendra \\ Babu, ${ }^{5}$ Dr Puneet Kumar Aggarwal, ${ }^{6}$ E. Fantin Irudaya Raj \\ ${ }^{1}$ Professor, Department of Computer Science and Engineering, KCG College of Technology, \\ Karapakkam, Chennai- 600 097, Tamilnadu India, dhanalakshmisaicse@outlook.com \\ ${ }^{2}$ Associate Professor, Department of Electronics and Communication Engineering, RVR and JC \\ College of Engineering, Chowdavaram, Guntur - 522019, d.e.chaitanya@ gmail.com \\ ${ }^{3}$ Associate Professor, Department of Computer Science and Engineering, CMR College of \\ Engineering \& Technology, Hyderabad, Telangana, India, chandu.pundru@ gmail.com \\ ${ }^{4}$ Professor \& Vice Principal, Department of Electronics and Communication Engineering, \\ Aarupadai Veedu Institute of Technology, Vinayaka Mission's Research Foundation, \\ Paiyanoor-603 104, Tamil Nadu, India, drdvijendrababu@ gmail.com \\ ${ }^{5}$ Assistant Professor, Department of Information Technology, ABES Engineering college, \\ Ghaziabad, Uttar Pradesh - 201009, puneetaggarwal7@gmail.com \\ ${ }^{6}$ Assistant Professor, Department of Electrical and Electronics Engineering, Dr. Sivanthi \\ Aditanar College of Engineering, Tamil Nadu- 628215, India, fantinraj@gmail.com
}

\begin{abstract}
The satellite communication is embellished constantly by providing information, ensuring security, and enables the communication among huge at a particular time efficiently. The satellite navigation helps in determining the people's location. The global development, natural disasters, change in climatic conditions, agriculture crop growth, etc. are monitored using satellite observation. Hence, the satellite includes detailed information data, it has to be protected confidentially. The field of the satellite is enhanced at an astonishing pace. Satellite data plays an important role in this modern world and hence, the onboard satellite data have to secure through the proper selection of error detection and estimation schema. The Extended Kalman Filter is widely used in the satellite system. EKF is utilized in this proposed model to detect the onboard pointing error such as attitude and orbit determination. An autonomous estimation of orbit position is possible through space-borne gravity. The information obtained through the observation of satellite data is compared with the accurate gravity model in detecting the error. The utilization of EKF reduces the dependence of the ground tracking system in satellite determination. The orbital altitude and orbital position are the most important challenges faced in the satellite determination system. The satellite model using the Extended Kalman Filter is a more apt method in estimating the orbital parameters. The errors in the linearization process are detected and this can be overcome
\end{abstract}


through the proper selection of linear expansion point with EKF algorithmic model with the Jacobian matrix calculation. The results show that the EKF implementation helps in attaining better accuracy in comparison with other methodologies.

\section{Keywords}

Extended Kalman Filter, Jacobian matrix calculation, Linear expansion point, Onboard pointing error, Satellite data, System nonlinear degree.

\section{INTRODUCTION}

In recent times, the global telecommunication system is more dependent on satellite communication. The satellite is more important in a contemporaneous application such as longdistance cellular calls, radio, and cable television. The satellite design includes the Global Positioning System (GPS) which provides the information regarding the present location and helps in directing us to the place we want to go. Satellite communication is a wide area of communication that covers a huge area within one satellite. Satellite are usually of two types manmade and natural. Earth and moon are considered to be the natural satellite whereas the manmade satellite is machines that are launched into space and orbits around a body in space. The source error in GPS is atmospheric interference, calculation and rounding errors, ephemeris data error, and multi-path effects.

The error detection and estimation in the satellite are performed using more algorithmic methods namely Extended Kalman Filter (EKF), Unscented Kalman Filter (UKF), and particle filter ([1], [2], and [3]). In the contemporaneous applications, the aptest algorithmic method in error detection and estimation is Extended Kalman Filter (EKF) [4]. The features which make the EKF practically suitable are easy implementation, reduced complexity during computation, and requires less hardware [5]. The aptest method is EKF which is preferably used in the implementation of satellite projects. Alsat-1, SNAP-1, UoSAT are some of the onboard-satellite computers which work under normal conditions ([6] and [7]). Though EKF has more advantages, it is unable to adjust itself as per the sensor uncertainty. The faulty measurement in the satellite may affect its estimation precision and thus, satellite navigation is affected by catastrophes.

The algorithmic model is also developed for the accurate determination of the Low Earth Orbit (LEO) [8]. In this algorithmic model, IGS orbits and accurate clock are used for the GPS satellite. The difference obtained between the code derived position and phase derived position helps in identifying the position of the satellite. The satellite position and processing speed can be monitored through the positioning of the orbit model in the desired position. The orbit model is fitted depending on the least square adjustment that requires the pseudo-observations. The pseudoobservation is formed by the combination of estimated position from both the code observation and phase observation. Unscented Kalman filter (UKF) is utilized in the development of an onboard orbit determination algorithm to satisfy the space-borne GPS receiver applications [9]. In onboard processing, accurate orbit navigation is attained through the employment of geopotential, 
atmospheric drag, the pressure of solar radiation, and the gravity of the Sun and Moon. The propagation of orbit is measured through the theoretical calculation method namely the RungeKutta method. The orbit motion is estimated through the implementation of the Cowell method. The position of the orbit in an artificial satellite could be determined using the least square algorithm where this algorithmic model includes sequential rotation and GPS receiver's data for the estimation purpose [10]. The Extended Kalman Filter (EKF) and the GPS forms an algorithmic model to determine onboard orbit in a satellite. This algorithmic model is simple and compact and hence, its computational cost is very low. The state vector, bias, GPS receiver clock drift rate, position and velocity composition, and drift are determined through the utilization of Extended Kalman Filter (EKF). The onboard error detection and estimation in the satellite are effective in the case of the Extended Kalman Filter (EKF) algorithmic model [11]. The accuracy level of tens of meters is consisting of the least value of velocity, position, and user clock offset components. The evaluation of the proposed dynamic model undergoes several testings, covering the force model, simplified variation equations, and the numerical integration and step size. The accurate algorithmic model includes a GPS navigation sensor that provides the information regarding the navigation process to determine the LEO satellite orbit.

\section{DISCUSSION}

The dynamic model can determine the orbit. The system model is more stable and provides accurate information regarding the orbital position [12]. The observation helps in estimating the error and helps in determining the geometrical orbit. This is more challenging as the model requires accuracy in observation and hence, it is a difficult task that traces the accurate orbit position [13]. The association of dynamical state and the information gathered from the geometric observation results in the development of kinematic orbit estimation. This kinematic method based on the observation quality can provide the accurate orbit computation point [14]. The drawbacks mentioned above can be overcome by the proposed reduced dynamic orbit determination. The target can be achieved by the geometric measurement and dynamic force model which consists of sequential filtering ([15] and [16]).

The conventional orbit determination has powerful ground computing with the available groundbased tracking data. Many tracking stations are set up to provide the information from the observed data and the gathered information is sent to the International GNSS Service (IGS) to develop three different types of orbital products namely ultra-rapid orbit, rapid orbit, and final orbit. These orbital products can achieve accuracy [17]. When the observation length is small or troposphere delay is severe, then the accuracy of the ground geometric measurement method is poor. The processing system based on the ground-based data is not highly secured. In the case of natural calamities such as water disasters and earthquakes, ground devices are affected [18]. An autonomous satellite navigation system developed in space generates information regarding orbit position and the intersatellite velocity which is independent of ground-based support. 
Autonomous space-based satellite navigation has improved reliability and stability [19]. The accurate orbit determination is achieved through the implementation of satellite navigation as it provides very precise information than the ground-based support [20]. Though the complexity is more in the satellite navigation, the benefits are also simultaneously high. The complex functional features such as fixed the transceivers in the satellite can send and receive signals. The programs are inbuilt in the satellite to perform automatically. High computing potential, high energy, and high reliability are required for the autonomous performance of satellites. If there is no availability of tracking data, then the autonomous navigation faces difficulty in orbit measurement. During the analysis of the orbit parameter, rotational error may result in rank defect if it is not esteemed [21].

The batch processing mode is the aptest conventional satellite navigation system for data smoothing. The orbit estimation process can be performed by collecting a huge number of data and these data are even suitable for post-processing. This conventional satellite navigation consists of computing and storage resources to satisfy the space-based environment. The sequential method is far better in comparison with the batch processing mode as this method generates the new observation for the accurate orbit determination. This method requires a very low computing capability and less memory. Thus, for the implementation of autonomous satellite navigation, this sequential method is mostly preferred as it requires fewer hardware resources and battery power. The filtering algorithms include the sequence processing is well known as the Kalman filter.

The recursive Kalman filter is the most effective in determining the internal state of the proposed system. The Kalman filter is more apt for the linear system whereas the contemporaneous application such as orbit determination comes under the non-linear satellite navigation systems [22]. The most familiar method in the filtering method is Extended Kalman Filter (EKF). The standardized linear recursive Kalman filter algorithm uses the Taylor series approximation [23]. The estimation of non-linear state, navigation systems, and GPS are determined by the Extended Kalman Filter (EKF) system model. The orbit determination in the contemporaneous is estimated through EKF. In EKF, during the kinematic calculation, the previously available state estimation is required. The orbit determination and autonomous satellite navigation are independent of the historical observation while increasing computational efficiency. In comparison with the traditional methods, EKF has the potential to predict accurately using the previous states. EKF forecast orbit determination along with the velocity satellite. Accurate orbit determination, satellite attitude coefficients ([24] and [25]), atmosphere, velocity, and clock are estimated using EKF.

Accuracy in determination can be achieved through the utilization of a reduced dynamic model based on the processor of the satellite. The stability of the filter algorithm is affected due to the error ignorance in the dynamic model. The error in the no-modeling system is compensated using the dynamic noise ([26] and [27]). Jacobian matrices along with the Extended Kalman Filter (EKF) implementation faced more challenges in some satellite systems. These challenges are solved through the development of the Unscented Kalman Filter (UKF). The big observations error, sample intervals, and large initial errors are overcome using UKF ([28] and [29]). Some other filter 
algorithms such as $\mathrm{H}_{\infty}$ filter and particle filter $(\mathrm{PF})$. The performance speed is high in $\mathrm{PF}$ in comparison with EKF and UKF [30].

In the case of satellite orbit determination, the EKF algorithm is the most preferred algorithmic model in the field of satellite [31]. The high-order truncation error is the difficulty faced due to the implementation of the first-order Taylor series in the EKF algorithm. The distinction observed from the processing model and practical model causes the linearization and approximation error. The nonlinear is the important feature in the proposed model. The higher the rate of nonlinear level, the higher will the error in the system model. EKF also depends on the difference between the linear expansion point and the original state. The divergence due to the inaccurate computation of the Jacobian matrix. These are consequence which results in filtering error over space.

\section{MATERIALS AND METHODS}

\section{System nonlinear degree:}

The system nonlinear degree is the important feature considered in the satellite determination. In the case of the nonlinear system model, the Taylor series expansion is used in the orbit determination. The system consists of many nonlinear characters that correspondingly increases the linearization error. An accurate linear model is achieved only with a zero-linearization error. The satellite orbital position is to keep on changing due to the nonlinear degree of force model. The elliptical orbit in a satellite consists of perigee and apogee. At the perigee and apogee point, the nonlinear degree is higher than at the orbital position. The perigee position faces more complexity due to drags. The precession of the orbit is caused by the apogee. Hence, the linearization error is larger at the perigee and apogee point.

\section{Linear expansion point:}

The time of the linear function and predicted time can be acquired under ideal conditions without any error. The orbit determination is not like its original state due to the random errors at the linear expansion point which results in a linearization error in a force model. If the linear expansion error increases, then the linearization error also increases. The linearization faces difficulty in attaining the accuracy of the linear expansion point. The point nearer to the original state must ignore the magnified error in an orbit determination task. Orbit elements, broadcast ephemerides, geometric approaches are some of the parameters considered in the determination process. The various determination approaches result in different accuracy rates. The satellite traction by ground-based and space-based stations faces challenges in fixing the initial orbital position. The filter approach is unstable when the big bias occurs periodically in the initialization phase. In case of any disaster, the satellite losing its orbital capability.

\section{Jacobian matrix calculation:}

The linearization applies to the non-linear system only when the Jacobian matrix calculation exists. The orbit determination is very challenging in the force model while executing practically and analytically using the Jacobian matrix. The Jacobian matrix must be implemented very carefully. The Jacobian matrix is implemented in converting the errors of a nonlinear variable space to 
linearized function space. The Jacobian matrix calculation is depending on the expansion point. The proper selection of expansion points is necessary for meeting the contemporaneous application of satellite. In the case of an improper Jacobian matrix, EKF is leading to an unstable and divergence system. During the filtering procedure, the complex influence is ignored. This algorithmic model has a very good performance evaluation.

Extended Kalman Filter (EKF): The attitude and orbit determination of the satellite is determined through the implementation of Extended Kalman Filter (EKF). In case of gyroscope malfunctions, this filter model is inconsiderable for measurements. The REKF is employed in this proposed system as follows [32],

The seven-dimensional state vector is given as follows,

$X=[q, \omega]^{T}=\left[q_{1}, q_{1}, q_{1}, q_{1}, \omega_{x}, \omega_{y}, \omega_{z}\right]^{T}$

\section{Step 1: Propagation cycle}

The numerical integration represents the dynamic states of the satellite.

$$
\begin{aligned}
& \overline{\mathrm{q}}_{\mathrm{k}}=\hat{\mathrm{q}}_{\mathrm{k}}+\frac{1}{2} \int_{\mathrm{t}_{\mathrm{k}}}^{\mathrm{t}_{\mathrm{k}+1}} \Omega\left(\omega_{\mathrm{k}}^{\circ}\right) \mathrm{q}_{\mathrm{k}} \mathrm{dt} \\
& \bar{\omega}_{\mathrm{k}}=\widehat{\omega}_{\mathrm{k}}+\int_{\mathrm{t}_{\mathrm{k}}}^{\mathrm{t}_{\mathrm{k}+1}}\left[\mathrm{I}_{\mathrm{s}}^{-1}\left(\mathrm{~T}_{\mathrm{GG}}+\mathrm{T}_{\mathrm{D}}-\mathrm{T}_{\mathrm{C}}-\omega_{\mathrm{k}} \times\left(\mathrm{I}_{\mathrm{S}} \omega_{\mathrm{k}}+\mathrm{h}\right)\right)\right] \mathrm{dt} \\
& \bar{X}_{\mathrm{k}}=\left[\begin{array}{l}
\overline{\mathrm{q}}_{\mathrm{k}} \\
\bar{\omega}_{\mathrm{k}}
\end{array}\right]
\end{aligned}
$$

The covariance matrix of the predicted error is given as follows,

$\overline{\mathrm{P}}_{\mathrm{k}}=\phi_{\mathrm{k}} \widehat{\mathrm{P}}_{\mathrm{k}} \phi_{\mathrm{k}}^{\mathrm{T}}+\mathrm{Q}_{\mathrm{k}}$

Here, The covariance matrix process is denoted as $Q_{k}$ and the state transition matrix is denoted as $\phi_{\mathrm{k}}$.

The state transition matrix is expressed as follows,

$\phi_{\mathrm{k}} \approx \mathrm{I}_{7 \times 7}+\left[\begin{array}{ll}\left.\frac{\partial \dot{\mathrm{q}}}{\partial \mathrm{q}}\right|_{\mathrm{t}=\mathrm{t}_{\mathrm{k}}} & \left.\frac{\partial \mathrm{q}}{\partial \omega}\right|_{\mathrm{t}=\mathrm{t}_{\mathrm{k}}} \\ \left.\frac{\partial \dot{\omega}}{\partial \mathrm{q}}\right|_{\mathrm{t}=\mathrm{t}_{\mathrm{k}}} & \left.\frac{\partial \dot{\omega}}{\partial \omega}\right|_{\mathrm{t}=\mathrm{t}_{\mathrm{k}}}\end{array} \mathrm{T}_{\mathrm{S}}\right.$

Here, $I_{7 \times 7}$ is the representation of identity matrix with $7 \times 7$ dimension and

The sampling period is expressed as $T_{S}=t_{k+1}-t_{k}$. 


\section{Step 2: Correction cycle}

The observation matrix is estimated as follows,

$\mathrm{H}_{\mathrm{k}}=\left[\begin{array}{cc}\frac{\partial \widetilde{\mathrm{B}}_{\mathrm{k}}}{\partial\left(\mathrm{q}_{1}, \mathrm{q}_{2}, \mathrm{q}_{3}, \mathrm{q}_{4}\right)} & \frac{\partial \widetilde{\mathrm{B}}_{\mathrm{k}}}{\partial\left(\omega_{\mathrm{x}}, \omega_{\mathrm{y}}, \omega_{\mathrm{z}}\right)} \\ \frac{\partial \widetilde{\omega}_{\mathrm{k}}}{\partial\left(\mathrm{q}_{1}, \mathrm{q}_{2}, \mathrm{q}_{3}, \mathrm{q}_{4}\right)} & \frac{\partial \widetilde{\omega}_{\mathrm{k}}}{\partial\left(\omega_{\mathrm{x}}, \omega_{\mathrm{y}}, \omega_{\mathrm{z}}\right)}\end{array}\right]$

The Kalman gain $\mathrm{K}_{\mathrm{k}}$ is tuned by introducing a noise scale factor in the filter is given as follows,

$\bar{s}_{\mathrm{k}}=\frac{\mathrm{e}_{\mathrm{k}}^{\mathrm{T}} \mathrm{e}_{\mathrm{k}}-\operatorname{tr}\left\{\mathrm{H}_{\mathrm{k}} \overline{\mathrm{P}}_{\mathrm{k}} \mathrm{H}_{\mathrm{k}}^{\mathrm{T}}\right\}}{\operatorname{tr}\{\mathrm{R}\}}$

Here, $e_{k}$ is the representation of residual term or innovation sequence.

The residual term is expressed as follows,

$\overline{\mathrm{e}}_{\mathrm{k}}=\left[\begin{array}{c}\widetilde{\mathrm{B}}_{\mathrm{k}} \\ \widetilde{\omega}_{\mathrm{k}}\end{array}\right]-\left[\begin{array}{c}\widehat{\mathrm{B}}_{\mathrm{k}} \\ \widehat{\omega}_{\mathrm{k}}\end{array}\right]$

The magnetometer value is determined with the $\widehat{B}=A(\widehat{q}) B^{\circ}$.

Here, the trace of the related matrix is represented as $\operatorname{tr}\{\cdot\}$.

The Kalman gain is determined through the following expression,

$\mathrm{K}_{\mathrm{k}}=\overline{\mathrm{P}}_{\mathrm{k}} \mathrm{H}_{\mathrm{k}}^{\mathrm{T}}\left(\mathrm{H}_{\mathrm{k}} \overline{\mathrm{P}}_{\mathrm{k}} \mathrm{H}_{\mathrm{k}}^{\mathrm{T}}+\overline{\mathrm{S}}_{\mathrm{k}} \mathrm{R}\right)^{-1}$

The expression for the estimation of covariance correction matrix is given as follows,

$\widehat{\mathrm{P}}_{\mathrm{k}}=\left(\mathrm{I}_{7 \times 7}-\mathrm{K}_{\mathrm{k}} \mathrm{H}_{\mathrm{k}}\right) \overline{\mathrm{P}}_{\mathrm{k}}$

Here, the corrected error covariance matrix is denoted as $\widehat{P}_{k}$ and the value of $R$ gives the noise measurement of the covariance matrix. Thus, the noise measurement of the magnetometer sensors and gyroscope is obtained.

The expression for the corrected state vector is given as follows,

$\widehat{\mathrm{X}}_{\mathrm{k}}=\left[\begin{array}{c}\hat{\mathrm{q}}_{\mathrm{k}} \\ \widehat{\omega}_{\mathrm{k}}\end{array}\right]=\overline{\mathrm{X}}_{\mathrm{k}}+\mathrm{K}_{\mathrm{k}} \overline{\mathrm{e}}_{\mathrm{k}}$

\section{RESULT AND DISCUSSION}

The performance of orbit determination is evaluated under the consideration of few features. The main feature important in determination is attaining accuracy. The comparison between the obtained state value and original state value generates the time-domain error curve. This timedomain error curve implies the accuracy of the filter. The consequence faced in the satellite navigation is estimated using the conditional covariance matrix. The accuracy of the prediction is also represented by this matrix. 
The variance of the filter is observed and predicted. The information regarding the state components is continuously gathered through the steady observation capability and hence, the occurrence of an error in state condition is estimated through the observation. The features such as sampling rate of data and measurement of noise level are responsible for affecting the steady-state variance. The convergence speed of the filter generates information about the performance of the filter which is inferred during observation.

The extended Kalman filter (EKF) is a recursive filter that is effective in the determination of the internal state from noisy measurement series. It only requires an estimation of the previous state for performing its calculation. From the results obtained and the graphs plotted, it is clearly shown that the extended Kalman filter with the improvements using the Jacobian matrix calculation point showed fewer truncation errors at a higher order and also increased the orbit accuracy significantly.

The relationship between the approaching line and the nonlinear curve is shown in figure 1 . The point of intersection of the approaching line and the nonlinear curve is at point $\mathrm{D}$. The point $\mathrm{D}$ shows the function of the true state $x^{\prime} k$. The true state $x k$, the function of $x k$ is given as $f(x k)$ at point $B$ in the nonlinear curve and the function at the same true state is given as $f^{*}(x k)$ point $A$ in the approaching line. The difference between the approaching line and the nonlinear curve is given by the $\overline{A B}$.

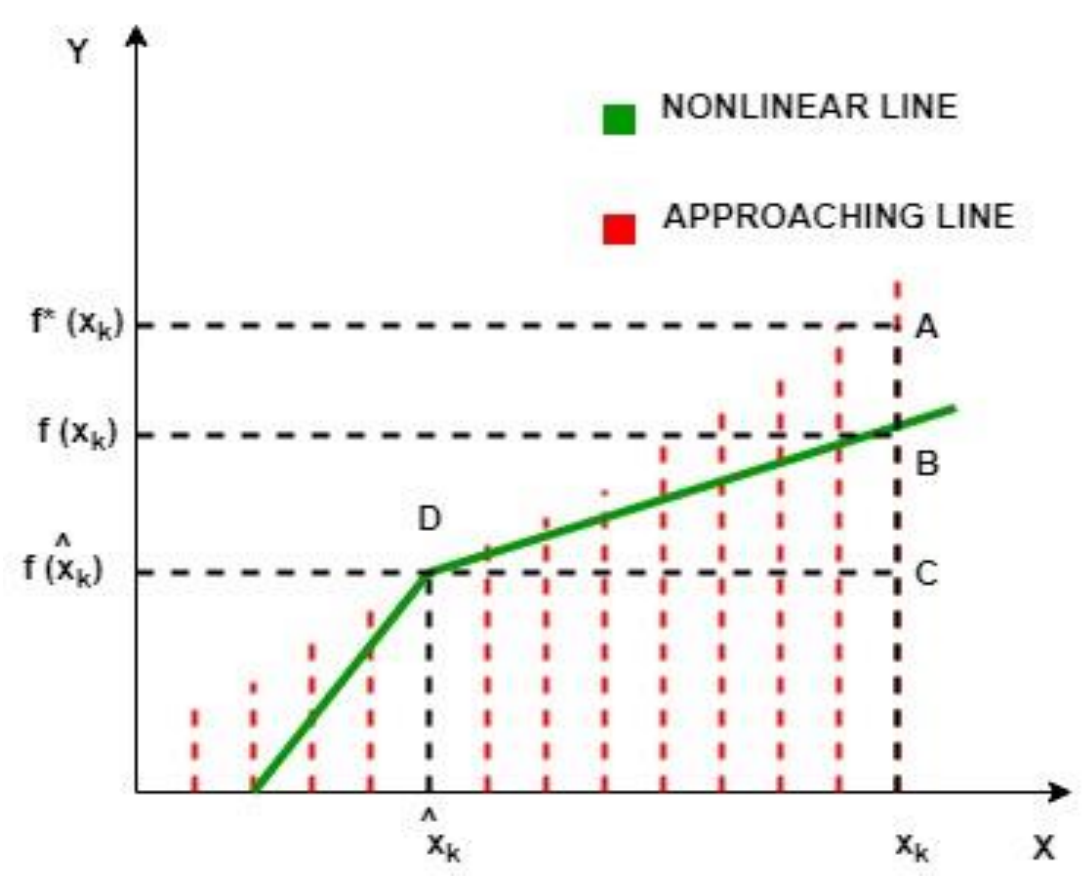

Figure 1: The relationship between nonlinear curve and approaching line. 
The approaching line in the figure 2 is obtained from the explosion point. The explosion point is placed at the estimation level endpoint. This point is used in obtaining the approaching line. In the Jacobian matrix calculation, the explosion point calculation is randomly chosen from the interval of estimation. Thus the true state $x k$, the function of $x k$ is given as $f(x k)$ at point $B$ in the nonlinear curve and the function at the same true state is given as $\mathrm{f}^{*}(\mathrm{xk})$ point $\mathrm{A}$ in the approaching line. The difference between the approaching line and the nonlinear curve is given by the $\overline{A B}$.

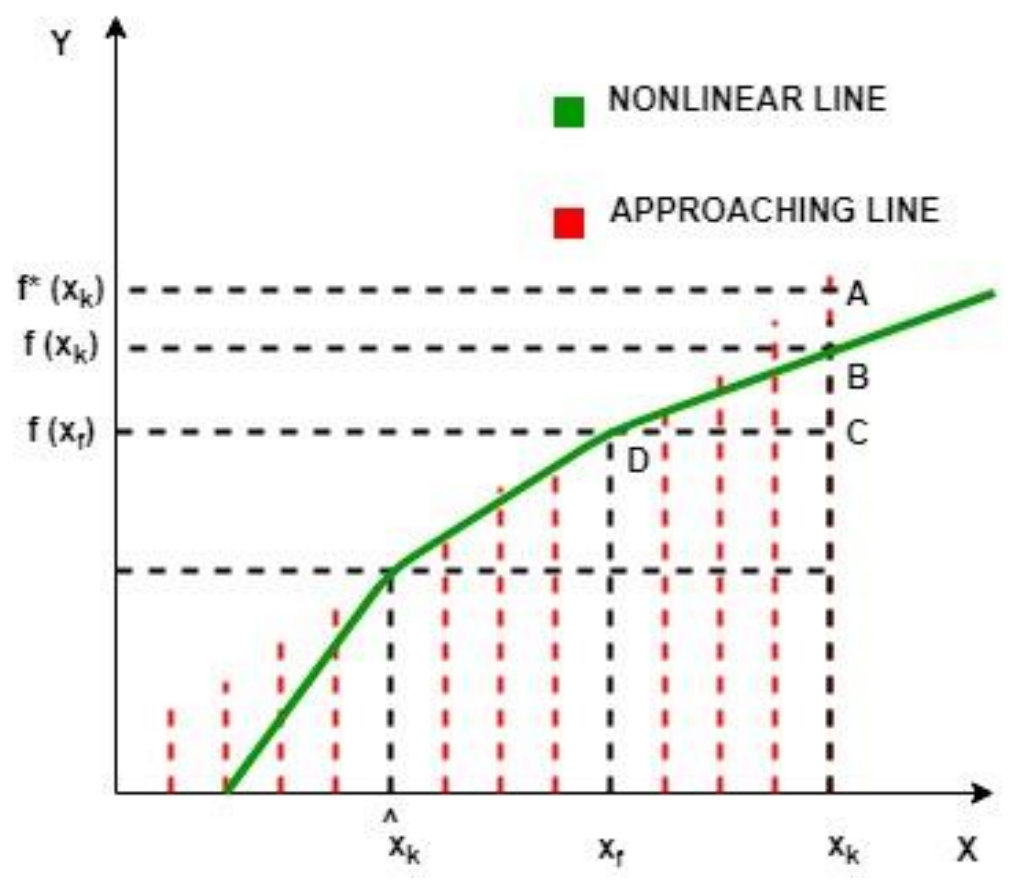

Figure 2: Linearization error when the explosion point is at the estimated interval. 


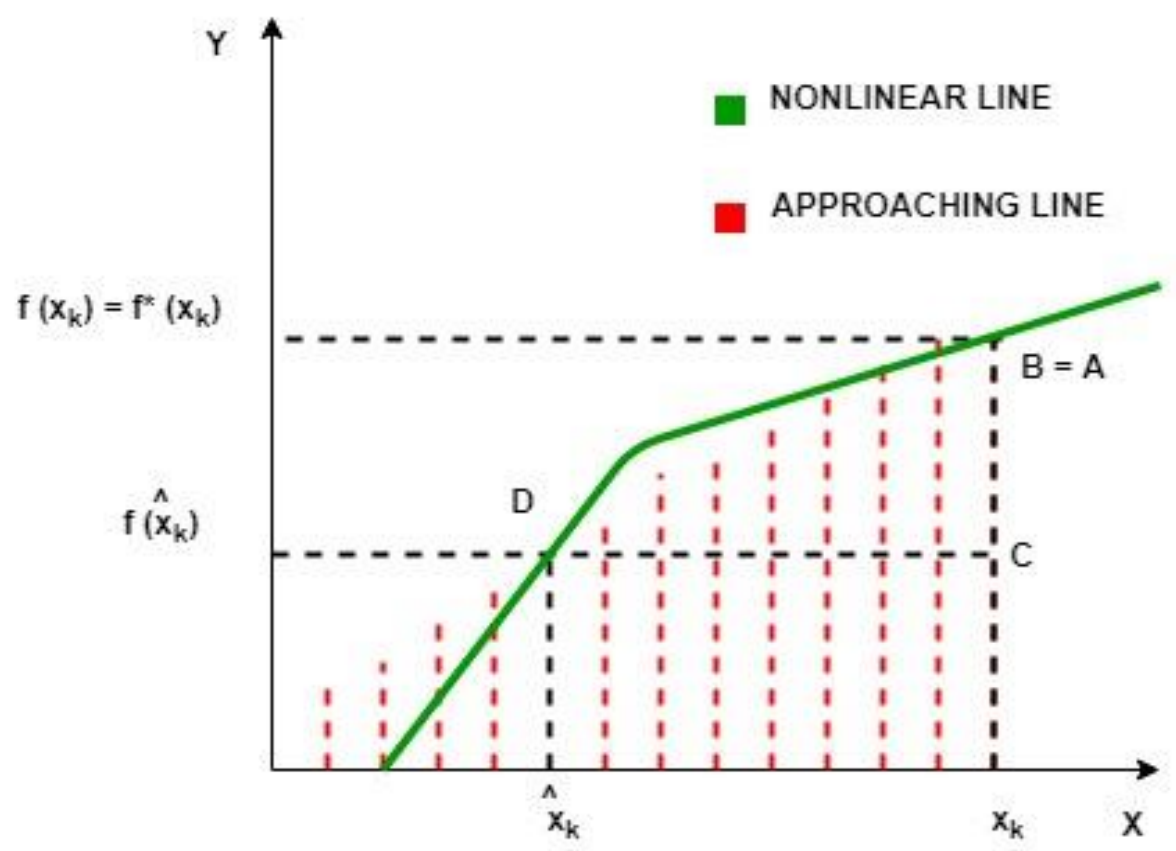

Figure 3: The approaching line is acquired when the explosion point is fixed.

The endpoint of the interval is taken as the explosion points is shown in figure 3 . The endpoints of the computing interval are used for the Jacobian matrix calculation point. Thus the explosion points are used in the process of obtaining the approaching line. Thus the true state $x k$, the function of $x k$ is given as $\mathrm{f}(\mathrm{xk})$ at point $\mathrm{B}$ in the nonlinear curve and the function at the same true state is given as $f^{*}(\mathrm{xk})$ point $\mathrm{A}$ in the approaching line. In the above curve, both the points overlap, and thus the difference between both the points becomes zero. Thus this makes clear that the approaching line error due to truncation becomes nil. 


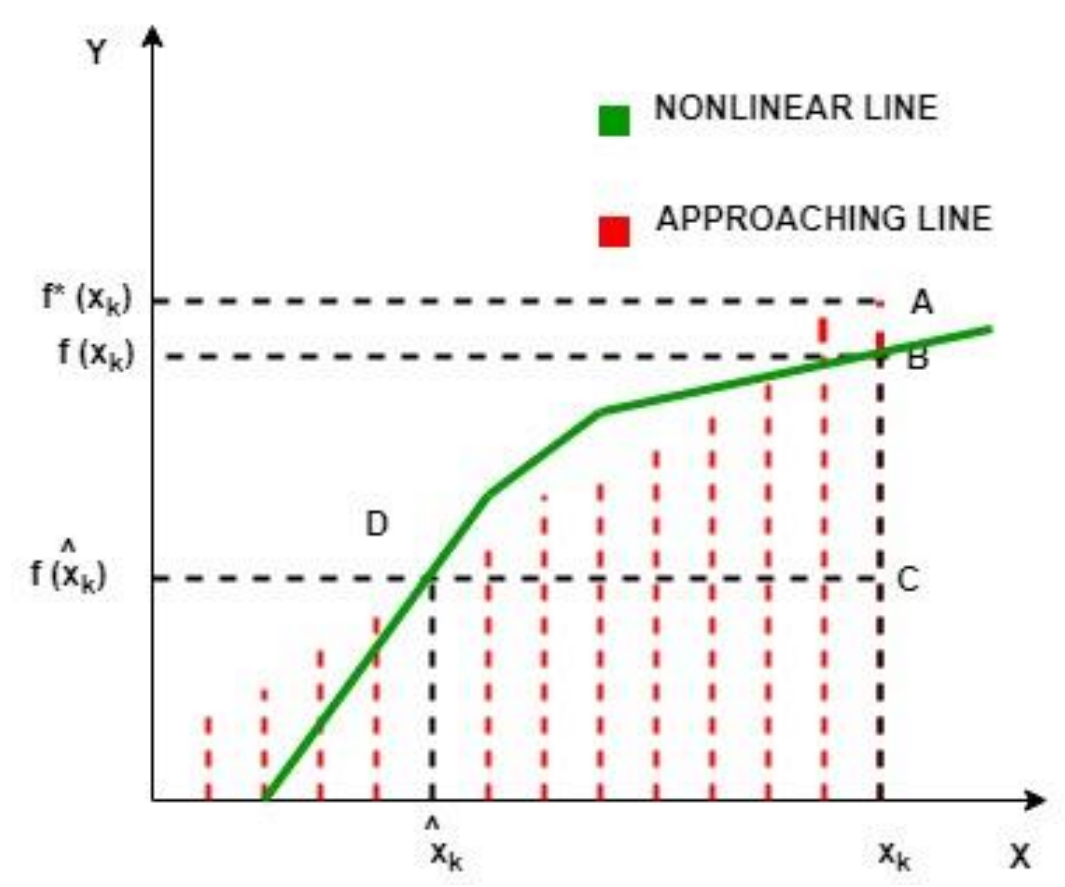

Figure 4: The linearization error at the estimated interval is very small.

The figure 4 includes the estimation interval is chosen for both the explosion point and also for the Jacobian matrix calculation point. the true state $x k$, the function of $x k$ is given as $f(x k)$ at point $B$ in the nonlinear curve and the function at the same true state is given as $\mathrm{f}^{*}(\mathrm{xk})$ point $\mathrm{A}$ in the approaching line. In the above curve, both the points overlap and thus the difference between both the points are less. Thus, the error due to the truncation and localization is found to be very low.

\section{CONCLUSION}

In this proposed system model, onboard pointing error such as an error in attitude and orbit determination is detected and estimated through the development of an estimation algorithm based on the Extended Kalman Filter (EKF) with the Jacobian matrix calculation. The field of the satellite is enhanced at an astonishing pace as the data available in the satellite is more valuable and sensitive. Thus, there is a demand for error detection, and estimation is essential to protect the satellite data. The satellite determination system is mainly based on the gyroscope partial failure during the estimation. The Extended Kalman Filter (EKF) algorithmic model is examined in predicting the error in the satellite system. The error in the onboard pointing is estimated accurately with the better filtering performance. The Extended Kalman Filter (EKF) is utilized as a backup determination system to protect the microsatellite mission. The nonlinearity, observation noise, and initial condition errors are some of the errors occurring at the linear approximation. The proper selection of linear expansion points enables the performance of the linearization process. The EKF 
satellite determination system has a higher accuracy rate and strong reliability. This shows that EKF is the aptest method for contemporaneous orbit determination. In the future, Extended Kalman Filter (EKF) will be utilized for the development of autonomous satellite navigation. Hence, the LEO satellite determination is better analyzed using the improvised model.

\section{Declaration}

\section{Funding}

Not Applicable for this article

\section{Conflicts of interest/Competing interests.}

The authors declare that they have no conflict of interest.

\section{Availability of data and material}

Not Applicable for this article

\section{Code availability}

Not Applicable for this article

\section{REFERENCES}

[1] Y. Yafei, L. Jianguo, "Particle filtering for gyroless attitude/angular rate estimation algorithm", In Pervasive Computing Signal Processing and Applications (PCSPA), 2010 First International Conference on (pp.1188-1191) IEEE.

[2] R. A. J. Chagas, J. Waldmann, "Nonlinear filtering in a simulated three axis satellite attitude estimation and control tested", 2010, Journal of Aerospace Engineering, Sciences and Applications, 2(2), 37-49.

[3] Z. H. Mohd, A. Amran, H. A. Abu et al., "Review on attitude estimation algorithm of attitude determination system”, 2016, ARPN Journal of Engineering and Applied Sciences, Vol. 11, no.7.

[4] E. J. Lefferts, F. L. Markley, M. D. Shuster, "Kalman filtering for spacecraft attitude estimation", 1982, Journal of Guidance, Control, and Dynamics, 5(5), 417-429.

[5] B. Chen, Y. Geng, X. Yang, "High precision attitude estimation algorithm using three star trackers", 2012, In Intelligent Control and Automation, $201210^{\text {th }}$ World Congress on (pp. 4168-4173). IEEE.

[6] W. H. Steyn, Y. Hashida, V. Lappas, "An attitude control system and commissioning results of the snap-1 nanosatellite", 2000. 
[7] A. S. Mohammed, M. Benyettou, A. Boudjemai et al, "Seven state Kalman filter for LEO microsatellite attitude determination", 2010, Recent Advances in Signal Processing, Robotics, and Automation, 151-157.

[8] H. Bock, U. Hugentobler, T. Springer, and G. Beutlerl, "Efficient precise orbit determination of LEO satellites using GPS”, Advances in Space Research, 30(2), pp. 259-300, 2002.

[9] E. Choi, J. Yoon, B. Lee, S. Park, K. Choi, "Onboard orbit determination using GPS observations based on the unscented Kalman filter", Advances in Space Research, 46, pp. 1440-1450, 2010.

[10] P. Pardal, H. Kuga, and R. deMoraes, "Implications of the applications of recursive least squares algorithms to satellite orbit determination using GPS measurements", WSEAS Transactions on System, vol. 8, no. 3, pp. 334-343, 2009.

[11] A. Chiaradia, H. Kuga, and A. Prado, "Onboard and Real-Time Artificial Satellite Orbit Determination Using GPS”, Mathematical Problems in Engineering, Hindawi Publishing Corporation, 2013.

[12] R. Scharroo and P. Nicolaas Visser, "Precise orbit determination and gravity field improvement for the ERS satellites", J Geophys Res Atmos 1998; 103: 8113-8128.

[13] F. Eisenhauer, R. Schodel, R. Genzel, et al., "A geometric determination of the distance to the galactic center", Astrophys J Lett 2003; 597: 597-610.

[14] D. Svehla and M. Rothacher, "Kinematic precise orbit determination for gravity field determination", A window on the future of geodesy, Int Assoc Geod Symp 2005; 128: 181 188.

[15] O. Montenbruck, T. Van Helleputte, R. Kroes, et al., "Reduced dynamic orbit determination using GPS code and carrier measurements”, Aerosp Sci Technol 2005; 9: 261-271.

[16] D. Svehla and M. Rothacher, "Kinematic and reduced dynamic precise orbit determination of low earth orbiters", Adv Geosci 2003; 1: 47-56.

[17] R. Dach, E. Brockmann, S. Schaer, et al., "GNSS processing at CODE: status report", J Geod 2009; 83: 353-365.

[18] X. Li, M. Ge, X. Zhang, et al, "Real-time high-rate co-seismic displacement from ambiguityfixed precise point positioning: application to earthquake early warning", Geophys Res Lett 2013; 40: 295-300.

[19] MP Ananda and H. Bernstein, "Global positioning system (GPS) autonomous navigation", New York: Inst. of Electrical and Electronics Engineers Position Location and Navigation Symposium, 1990. 
[20] A. Rajan John, "Highlights of GPS II-R autonomous navigation. In: Proceedings of the $58^{\text {th }}$ Annual Meeting of the Institute of Navigation and CIGTF $21^{\text {st }}$ Guidance Test Symposium, Hyatt Regency Hotel, Albuquerque, NM, USA, 24-26 June 2002.

[21] W. Wang, X. Dong, W. Liu, et al., "Influence of satellite-to-ground link on the autonomous navigation of navigation constellation", In: China Satellite Navigation Conference (CSNC) 2012 proceedings, Guangzhou, China, Lecture notes in electrical engineering, vol. 160, Springer-Verlag Berlin Heidelberg, 2012, pp. 337-348.

[22] RE Kalman, “A new approach to linear filtering and prediction problem", Trans ASME 1960; 82: 35-45.

[23] Y. Sunahara, "An approximate method of state estimation for nonlinear dynamical systems", J Basic Eng Trans ASME Ser D 1970; 92: 385-393.

[24] A. Reichert, T. Meehan, and T. Munson, "Toward decimeter-level real-time orbit determination: a demonstration using the SAC-C and CHAMP Spacecraft", In: Proceedings of the $15^{\text {th }}$ International Technical Meeting of the Satellite Division of the Institute of Navigation (ION GPS 2002) 24-27 September 2002, Oregon Convention Center, Portland, OR, USA, pp. 24-27.

[25] Q. Zhang, P. Moore, J. Hanley, et al, "Auto-BAHN: soft-ware for near real-time GPS orbit and clock computations”, Adv Space Res 2007; 39: 1531-1538.

[26] X. Li, M. Ge, H. Zhang, et al., "A method for improving uncalibrated phase delay estimation and ambiguity-fixing in real-time precise point positioning", J Geod 2013; 87: 405-416.

[27] D. Joseph Mook and JL Junkins, "Minimum model error estimation for poorly modeled dynamic systems", J Guid Control Dyn 1988; 11: 256-261.

[28] SJ. Julier and JK. Uhlmann, "Unscented filtering and nonlinear estimation”, Proc IEEE 2004; 92: 401-422.

[29] EJ Choi, JC Yoon, BS Lee, et al., "Onboard orbit determination using GPS observation based on the unscented Kalman filter", Adv Space Res 2010; 46: 1440-1450.

[30] X Ning and J Fang, "Spacecraft autonomous navigation using unscented particle filter-based celestial/Doppler information fusion”, Meas Sci Technol 2008; 19: 1-8.

[31] T. Kelecy, M. Jah, "Detection and orbit determination of a satellite executing low thrust maneuvers. Acta Astronaut 2010; 66: 798-809.

[32] H. E. Soken, C. Hajiyev, "REFK and RUFK development for pico satellite attitude estimation in the presence of measurement faults", In Recent Advances in Space Technologies (RAST), $5^{\text {th }}$ International Conference on (pp. 891-896). IEEE, 2011. 

Figures

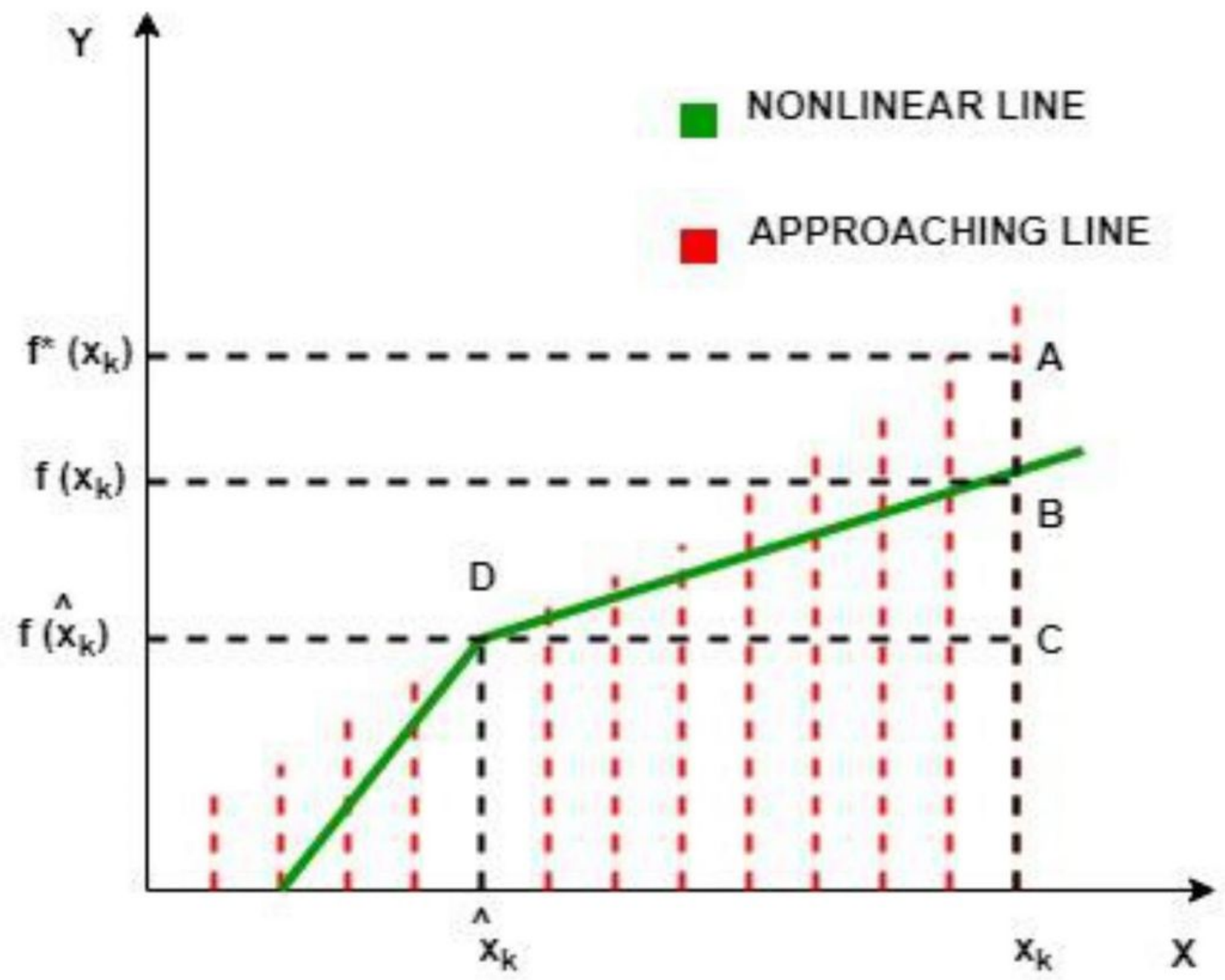

Figure 1

The relationship between nonlinear curve and approaching line. 


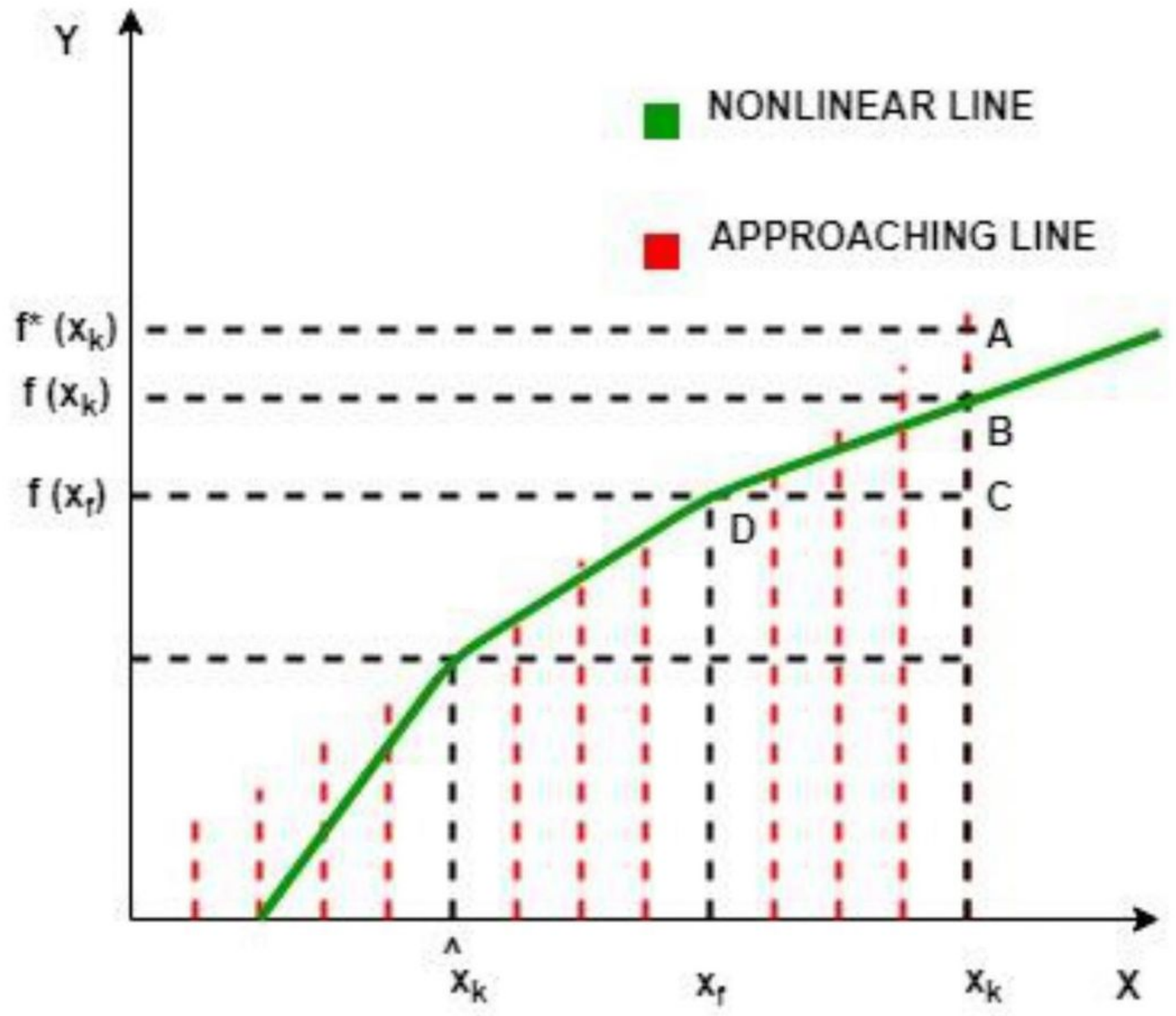

Figure 2

Linearization error when the explosion point is at the estimated interval. 


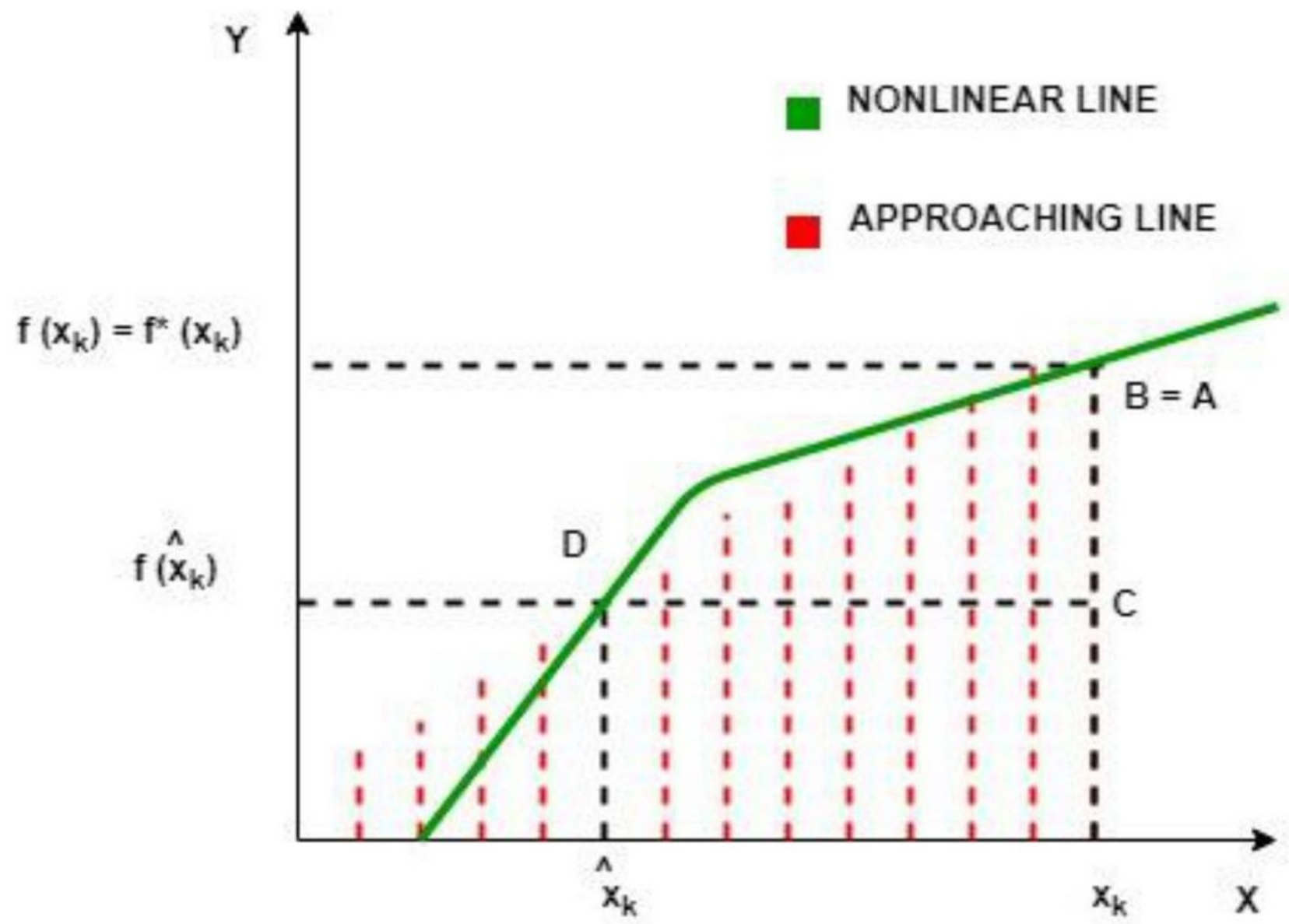

Figure 3

The approaching line is acquired when the explosion point is fixed. 


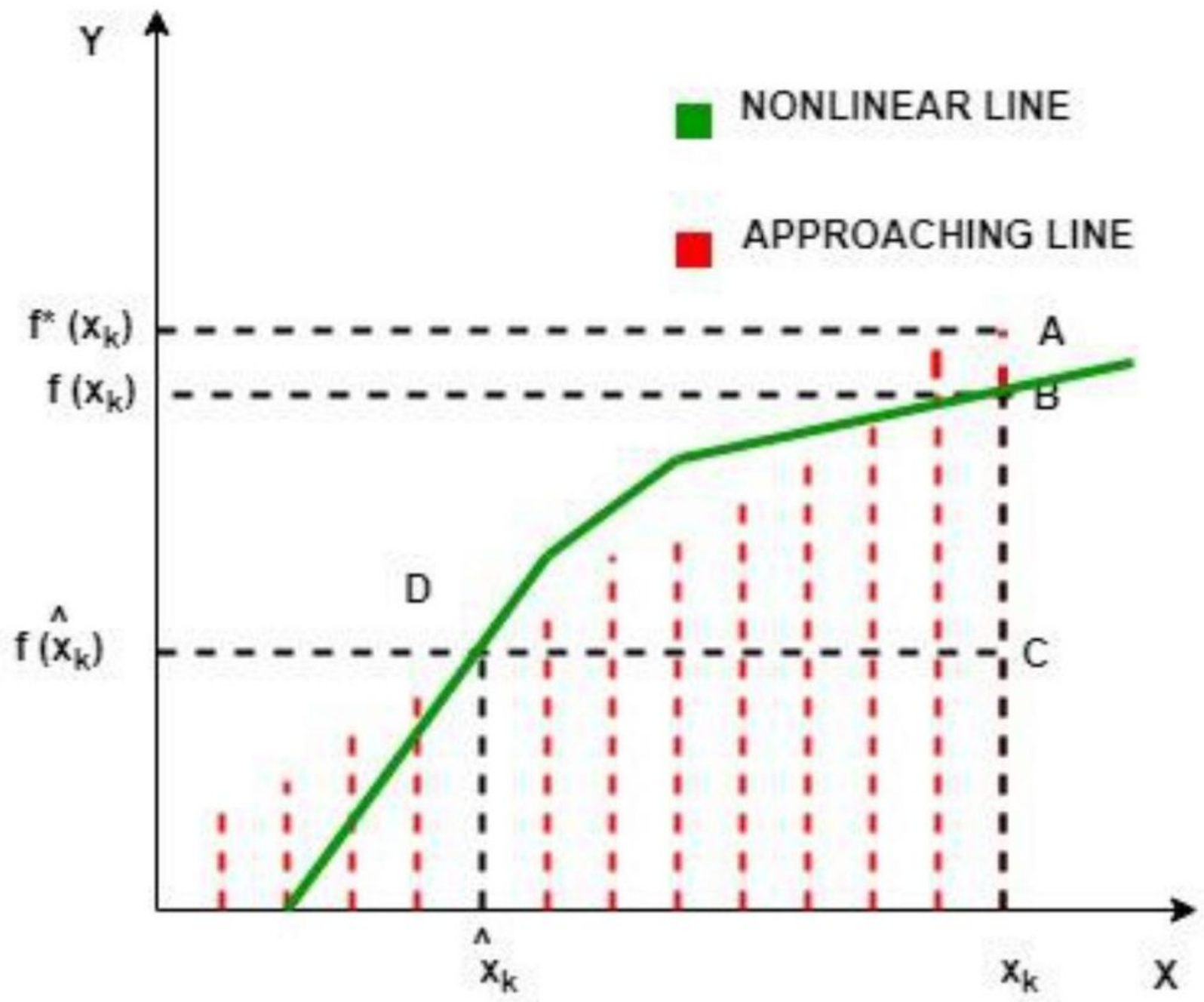

Figure 4

The linearization error at the estimated interval is very small. 